\title{
A comparison of cardiac magnetic resonance imaging peri-infarct border zone quantification strategies for the prediction of ventricular tachyarrhythmia inducibility
}

\author{
Jason C. Rubenstein ${ }^{1,2}$, Daniel C. Lee ${ }^{3,4}$, Edwin $\mathrm{Wu}^{3,4}$, Alan H. Kadish ${ }^{3}$, \\ Rod Passman ${ }^{3}$, David Bello ${ }^{5}$, Jeffrey J. Goldberger ${ }^{3}$ \\ ${ }^{1}$ Division of Cardiology, Department of Medicine, Medical College of Wisconsin, USA \\ ${ }^{2}$ Department of Radiology, Medical College of Wisconsin, USA \\ ${ }^{3}$ Division of Cardiology, Department of Medicine, Feinberg School of Medicine, \\ Northwestern University, USA \\ ${ }^{4}$ Department of Radiology, Feinberg School of Medicine, Northwestern University, USA \\ ${ }^{5}$ Mid-Florida Cardiology Specialists, Orlando, Florida
}

\begin{abstract}
Background: Peri-infarct border zone (BZ) as quantified by late gadolinium enhancement (LGE) on cardiac magnetic resonance imaging (MRI) has been proposed as a risk stratification tool, and is associated with increased mortality. BZ has been measured by various methods in the literature. We assessed which BZ analysis best predicts inducible arrhythmia during electrophysiological study (EPS).

Methods: LGE was performed in 47 patients with coronary artery disease referred for EPS to assess for ventricular tachycardia (VT). LGE data was analyzed for BZ quantification by 3 previously published methods. Method I (BZ-I) used pixels 2-3 standard deviations over the mean of normal tissue, expressed as \% of left ventricular mass, Method II (BZ-II, as described by Yan) and Method III (BZ-III, as described by Schmidt). EPS results were classified as negative (non-inducible) or positive (monomorphic VT-MVT).

Results: There were 47 subjects-age 61.7 years, 72\% male. During EPS, 20 patients were non-inducible and 18 had induced MVT. Ejection fraction was not significantly different between non-inducible patients and those with MVT (34.1\% vs. $28.5 \%, p=0.13)$. BZ-I was significantly different (1.4\% vs. $2.6 \%, p=0.001)$, but not BZ-II (7.9\% vs. $6.9 \%, p=0.68)$ or BZ-III (2.7 g vs. $2.1 \mathrm{~g}, p=0.88)$. Multivariate analysis demonstrated that only BZ-I was an independent predictor of EPS outcome after controling for infarct size (OR 1.97 per \% change, 95\% CI 1.04-3.73, $p=0.04$ ).

Conclusions: This study demonstrates significant variability between the published methods for measuring BZ. Also, BZ-I is a stronger predictor of inducible MVT during EPS than ejection fraction and infarct size. BZ may be another LGE marker of elevated risk of arrhythmia. (Cardiol J 2013; 20, 1: 68-77)
\end{abstract}

Key words: cardiac magnetic resonance imaging, ventricular tachycardia, sudden death

Address for correspondence: Jason Rubenstein, MD, Division of Cardiovascular Medicine, Froedtert East Clinics, 9200 West Wisconsin Avenue, Milwaukee, WI 53226, USA, tel: (414) 456-6777, fax: (414) 456-6203, e-mail: jrubenstein@mcw.edu 


\section{Introduction}

Sudden cardiac death (SCD) remains a pervasive modern health concern, with over 300,000 deaths annually in the United States alone [1]. Implantable defibrillators have proven to be an effective treatment modality in selected patients with coronary artery disease (CAD) [2-5]. The most widely used risk identifier for determining patients at risk for SCD is left ventricular ejection fraction (LVEF) [6]. While an effective parameter, LVEF has limited sensitivity and specificity; therefore identifying individuals at risk of SCD after myocardial infarction (MI) continues to be a challenging problem $[7,8]$. SCD in patients with CAD is predominantly caused by ventricular tachycardia (VT) or ventricular fibrillation (VF) $[4,9]$. The anatomic substrate defined by the infarction is a main component in the pathogenesis of these arrhythmias. Given cardiac magnetic resonance imaging's (MRI) ability to delineate the infarction [10], infarct characterization by cardiac MRI (CMR) has become an evolving novel method for risk stratification [11-14].

Infarct size is a known determinant of the risk for occurrence of VT [15-17]. Recent human studies have demonstrated that infarct characterization by late gadolinium enhancement (LGE) on CMR is predictive of overall mortality [12] as well as inducibility of ventricular arrhythmia during electrophysiological study (EPS) $[11,18]$. The peri-infarct border zone (BZ) has been defined histologically as the area of viable myocardium immediately adjacent to infarcted myocardium. This region has previously been shown in animal models to identify areas of abnormal enzyme activity, as well as altered electrical activation properties [19, 20]. Recently, "grey zone" imaging, has been introduced as a method for identifying the BZ by LGE and has been associated with increased mortality in a human population [14]. In delayed-enhanced CMR images, normal myocardium appears black and infarct white. The authors postulated that pixels with intermediate (grey) intensity identify a potentially arrhythmic heterogeneous zone of viable and nonviable peri-infarct myocardium, but this has not been confirmed pathologically. If this indeed represents the substrate for reentrant ventricular arrhythmias, there should be increased $\mathrm{BZ}$ among patients with inducible monomorphic VT (MVT) at EPS, a well accepted modality to demonstrate this substrate [21, 22]. Schmidt et al. [18] did identify BZ (in grams) to be a predictor of inducible VT, but infarct size was not. Interestingly, the techniques used for BZ quantitation in these latter 2 studies differed [14, 18]. Based on these recent data, we evaluated the predictive value of $\mathrm{BZ}$ relative to infarct size in our previously reported study of CMR determined infarct size and its relationship to inducible VT [11]. We hypothesized that $\mathrm{BZ}$ quantification by LGE would be more predictive of inducible arrhythmia during EPS than infarct size and ejection fraction.

\section{Methods}

This study was approved by the Northwestern University Institutional Review Board. This was a retrospective reanalysis of data from a prior study [11], in which 48 patients, all with known history of either chronic $\mathrm{CAD}$ or distant $\mathrm{MI}$, were referred for EPS to assess for inducibility of VT for primary prevention risk stratification of sudden death. No patients had prior history of sustained ventricular arrhythmias.

In this study, these LGE data were re-analyzed for $\mathrm{BZ}$ quantification. One patient's CMR could not be recovered and was excluded from this analysis. Patients underwent CMR within $32 \pm 6$ days of EPS, per the protocol of the prior study [11]. Patients were placed supine in a 1.5-T Magnetom Sonata scanner (Siemens, Medical Solutions, Malvern, Pennsylvania); fiberoptic electrocardiographic (ECG) leads were placed for scanner gating and a phased-array receiver coil was placed on the chest for imaging. All images were acquired using 10- to 15 -s breath-holds. Short-axis cines were acquired from the base to apex, making sure to include the entire left ventricle (LV) using methods previously described $[23,24]$. Typical CMR parameters were: matrix resolution 256, field-of-view 340 $-400 \mathrm{~mm} \times 225-380 \mathrm{~mm}$, slice thickness $6 \mathrm{~mm}$, voxel size $1.33-1.56 \mathrm{~mm} \times 1.56-2.43 \mathrm{~mm} \times 6 \mathrm{~mm}$. No parallel imaging was used. Repetition time (typically $59-60 \mathrm{~ms}$ ) was selected to be $100 \mathrm{~ms}$ less than the R-R interval, to place the acquisition window within end-diastole, with 23-25 segments per cardiac cycle. Delayed-enhanced images were obtained more than 10 min following intravenous administration of a gadolinium-based contrast agent $(0.2 \mathrm{mmol} / \mathrm{kg}$, Magnevist, Berlex Pharmaceuticals, Wayne, New Jersey) using a T1-weighted, inversion-recovery, segmented fast gradient-echo pulse sequence [25]. The inversion time was adjusted throughout the scan to null normal myocardium. The methods used for the quantification of LVEF, infarct size, and infarct surface area have all been previously described [11]. Briefly, endocardial and epicardial borders of the myocardium were manually planimetered on the short-axis cine images for 
each patient. Volumes were derived by summation of the pixel areas, followed by multiplication of inplane resolution and the effective slice thickness. The LVEF was computed as (end-diastolic volume - end-systolic volume)/end-diastolic volume. LV mass was determined by subtracting endocardial volume from epicardial volume at end-diastole and multiplying by a density of $1.05 \mathrm{~g} / \mathrm{mL}$ [18]. Infarct morphology was evaluated using the ceMRI images. The presence of MI, its location, and the degree of transmurality were agreed upon by two observers. To measure infarct mass and surface area, the infarct region was outlined according to whether the image intensity was $\geq 2$ SD that of a remote region in the same slice. From the contours, a pixel value was computed for the area and surface of each individual infarct territory. Based on the pixel values, the image resolution and slice thickness, and an assumed density of $1.05 \mathrm{~g} / \mathrm{mL}$, the pixel values were converted into actual cardiac masses and surface areas.

Electrophysiologic study was performed using standard techniques. Programmed ventricular stimulation was performed using up to three extrastimuli at two right ventricular sites during 2 drive-cycle lengths. Study end points were either induction of sustained VT or completion of the study protocol. EPS results had been previously classified as negative (no inducible ventricular tachyarrhythmia) $(n=20)$, inducible MVT $(n=18)$, or inducible VF or polymorphic VT (PVT) $(n=9)$. Because inducible PVT/VF is considered a nonspecific result during EPS, the primary analysis comparing LGE results, as well as comparing the different $\mathrm{BZ}$ calculation methods, evaluates the non-inducible group vs. those with inducible MVT, as in the original report. However, baseline characteristics and long-term outcomes are reported for all subjects.

Border zone has been previously quantified using two statistical techniques. Yan et al. [14] defined $\mathrm{BZ}$ by regions of signal intensity between 2 and 3 SD above normal myocardium. They then indexed this measure to the infarct size. Of note, using this index, there was an inverse relationship between infarct size and BZ (i.e. the larger the infarct, the smaller the BZ). Schmidt et al. [18] defined all pixels with signal intensity above the peak intensity in an area of normal myocardium and below $50 \%$ of the peak intensity in the infarcted region as $\mathrm{BZ}$. As neither definition has pathologic correlation, we analyzed the current data using both definitions. In addition, when LGE BZ was measured with the first method, we also indexed this to the total LV mass. As will be demonstrated, this restores the expected positive geometric relationship between infarct size and BZ (i.e. the larger the infarct, the larger the $\mathrm{BZ}$ ).

All image analysis was performed by a single investigator, blinded to the results of prior analysis, clinical outcomes and to EPS results. The methods for image analysis have been described in detail [26]. Briefly, all short-axis delayed-enhancement images from apex to base were reviewed offline using ImageJ (National Institute of Mental Health). A custom macro for ImageJ was written for the semi-automated quantification of LGE BZ. The macro-analyzes a single, post-contrast short axis image. The user first defines a region of LV remote from the infarct that appears normal. Next, epicardial and endocardial surfaces are defined. The macro generates a summation of all $\mathrm{LV}$ pixels that meet criteria for normal, BZ, or core infarct.

Due to significant variations in the published methods by which to calculate BZ, we next performed the BZ analysis by 3 different methods, which will be referred to as BZ-I, BZ-II, and BZ-III. Border zone-I was defined as all pixels in the endocardium that were between 2 and 3 SD above the mean of the normal region, and core infarct those above 3 . Border zone and core infarct were reported as a percentage of the total LV mass. Border zone-II was defined as in method I, but the values of $\mathrm{BZ}$ were reported as a percent of infarct size as described by Yan et al. [14] In BZ-III, BZ and core infarct mass were calculated using the full-width half-maximum method as described by Schmidt et al. [18]. For all methods, pixels between 2 and 3 SD above the mean of the remote region that were not adjacent to areas of core infarction ( $>3 \mathrm{SD}$ above remote mean) were not included in the $\mathrm{BZ}$ calculation. Two example studies are shown in Figure 1, comparing all 3 methods of BZ calculation.

Follow-up data on implantable cardioverter-defibrillator (ICD) shocks and mortality were assessed for all 47 patients by referencing the social security death index, as well as hospital and ICD chart review. ICD programming was done at the discretion of the treating physician; the average VT or VF zone cut-off was $167 \mathrm{bpm}$, range 95-188. Appropriate therapy was considered any high-energy shock or anti-tachycardia pacing for sustained ventricular arrhythmia.

\section{Statistical analysis}

Results are expressed as mean $\pm \mathrm{SD}$, unless otherwise noted. Discrete variables were compared across patient groups using the $\chi^{2}$, Fisher, or Spearman tests where appropriate. Continuous variables 


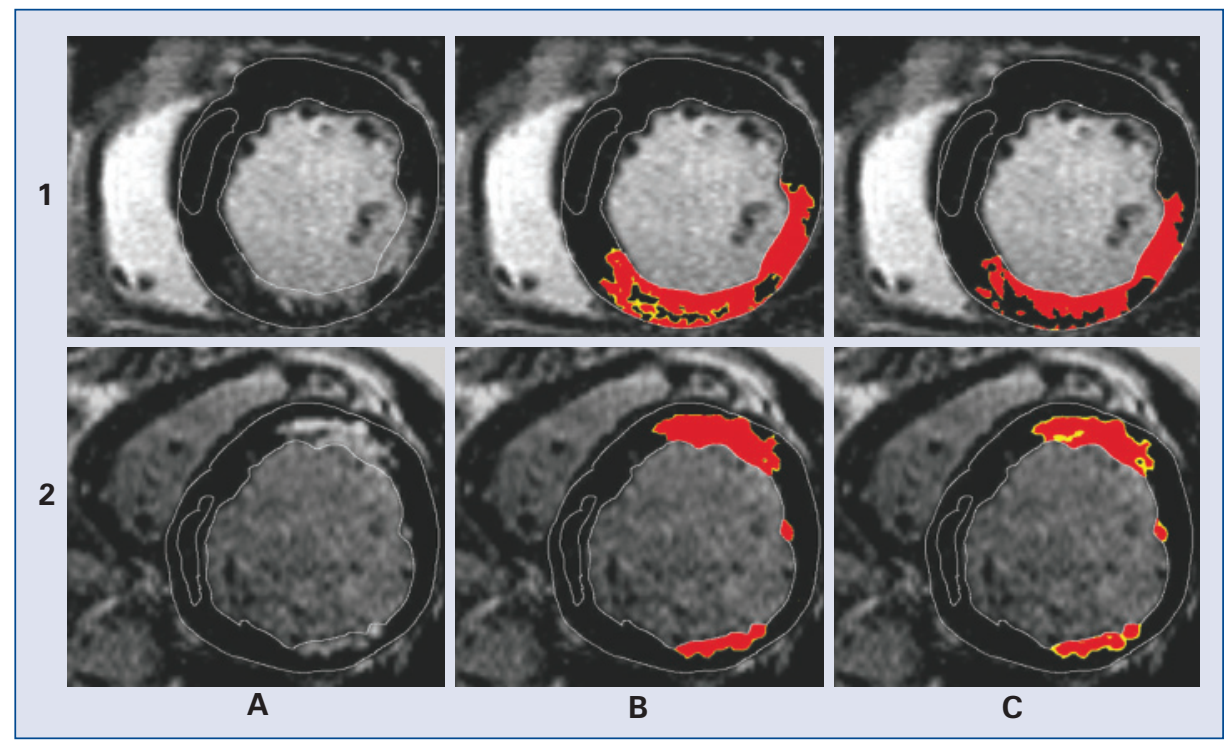

Figure 1. Example late gadolinium enhancement (LGE) images contrasting border zone (BZ) methods. Row 1 is a patient with a BZ of $2.5 \%$ by BZ-I, $11.2 \%$ by BZ-II, and $1.33 \mathrm{~g}(1.1 \%$ of left ventricular mass [LVM]) by BZ-III. This patient had an infarct size of $14.4 \%$, ejection fraction of $36.1 \%$, and was inducible for monomorphic ventricular tachycardia during electrophysiology study (EPS). Row 2 is a patient with a BZ of $0.8 \%$ by BZ-I, $4.1 \%$ by BZ-II, and $7.47 \mathrm{~g}(7.3 \%$ of LVM) by BZ-III. This patient had an infarct size of $15.4 \%$, ejection fraction of $18.3 \%$, and was noninducible during EPS. Column A shows the original magnetic resonance image with the endocardial, epicardial and normal region of interest identified in white. Column B demonstrates core infarct in red and BZ in yellow, as determined by BZ-I and BZ-II. Column C demonstrates core infarct in red and BZ in yellow, as determined by BZ-III.

were compared with Student's t-test, Kruskal-Wallis or Mann-Whitney tests, where appropriate. Analysis of non-parametric parameters (EF, infarct size, infarct surface area, LV mass, and BZ) utilize the Kruskal-Wallis test, and are reported as median value ( $1^{\text {st }}$ quartile, $3^{\text {rd }}$ quartile). Analysis of variance was performed to compare characteristics among all 3 EPS groups. Two separate multivariate logistical regressions were performed to identify independent determinants of EPS outcome for each method; one using $\mathrm{BZ}$ and infarct mass percentage alone, and one using $\mathrm{BZ}$, infarct mass percentage and LVEF. A 2-tailed p-value $<0.05$ was considered statistically significant. All analysis was performed using SPSS 16.0 (SPSS Inc, Chicago, IL). The authors had full access to the data and take responsibility for its integrity. All authors have read and agree to the manuscript as written.

\section{Results}

The study group consisted of 47 subjects with an average age of $61.7 \pm 11.4$ years and $72 \%$ were male. During EPS, 20 patients had no inducible ventricular arrhythmias, 9 had PVT or VF, and 18 had induced MVT. Baseline characteristics are shown in Table 1 . There were no significant differences among the three groups in terms of history of MI, congestive heart failure, diabetes mellitus, or beta-blocker and ACE inhibitor use. Only age, gender, and a reported history of hypertension were significantly different between the 3 EPS outcome groups. QRS duration on baseline 12-lead ECG was $110 \pm 6 \mathrm{~ms}$ in the non-inducible group, $121 \pm 5 \mathrm{~ms}$ in the MVT group, and $118 \pm$ $\pm 11 \mathrm{~ms}$ in the VF group, which was a non-significant difference.

\section{CMR results}

The differences in LGE results between the non-inducible and inducible EPS groups are shown in Table 2. As previously reported, LVEF was not significantly different between non-inducible patients and those with MVT (34.1\% vs. $28.5 \%, \mathrm{p}=0.13$ ), while infarct size $(15.8 \%$ vs. $23.1 \%, \mathrm{p}=0.03)$ and surface area $\left(104.4 \mathrm{~cm}^{2}\right.$ vs. $\left.169.8 \mathrm{~cm}^{2}, \mathrm{p}=0.002\right)$ were. No areas of microvascular obstruction were noted. All patients except 3 had some evidence of LGE ( 2 in non-inducible group, 1 in VF group). Analysis was repeated excluding these 3 patients, but no results were significantly altered. All results shown include these 3 patients. 
Table 1. Baseline characteristics

\begin{tabular}{lcccc}
\hline & $\begin{array}{c}\text { Total } \\
(\mathbf{n}=\mathbf{4 7})\end{array}$ & $\begin{array}{c}\text { Non-inducible } \\
(\mathbf{n}=\mathbf{2 0})\end{array}$ & $\begin{array}{c}\text { PVT/VF } \\
(\mathbf{n}=\mathbf{9})\end{array}$ & $\begin{array}{c}\text { MVT } \\
(\mathbf{n}=\mathbf{1 8})\end{array}$ \\
\hline Male & 26 & 10 & 8 & 16 \\
Age & $61.7 \pm 11.4$ & $59.3 \pm 11.9$ & $71.4 \pm 10.6$ & $61.3 \pm 9.7$ \\
History of MI & 25 & 12 & 4 & 13 \\
History of CHF & 26 & 12 & 4 & 14 \\
History of HTN & 19 & 8 & 1 & 11 \\
History of DM & 11 & 6 & 4 & 5 \\
History of HL & 20 & 7 & 8 & 13 \\
Beta-blocker & 30 & 16 & 6 & 14 \\
ACE-I & 32 & 15 & 4 & 17 \\
\hline
\end{tabular}

PVT — polymorphic ventricular tachycardia; VF — ventrifcular fibrillation; MVT — monomorphic ventricular tachycardia; MI — myocardial infarction; $\mathrm{CHF}$ - congestive heart failure; HTN — hypertension; DM — diabetes mellitus; HL — hyperlipidemia; ACE-I — angiotensin converting enzyme inhibitor

Table 2. Magnetic resonance imaging characteristics.

\begin{tabular}{lcccc}
\hline & $\begin{array}{c}\text { Total } \\
(\mathbf{n}=38)\end{array}$ & $\begin{array}{c}\text { Non-inducible } \\
(\mathbf{n}=\mathbf{2 0})\end{array}$ & $\begin{array}{c}\text { MVT } \\
(\mathbf{n}=\mathbf{1 8})\end{array}$ & P \\
\hline LVEF [\%] & $32.21(22.5,33.9)$ & $34.09(22.7,44.4)$ & $28.48(21.9,36.0)$ & 0.13 \\
Infarct size [\%] & $18.49(13.5,34.1)$ & $15.84(6.6,23.4)$ & $23.07(15.5,35.25)$ & 0.026 \\
Infarct SA [cm $\left.{ }^{2}\right]$ & $115.97(94.8,181.75)$ & $104.37(63.5,145.6)$ & $169.84(116.4,227.5)$ & 0.002 \\
LV mass [g] & $195.36(147.6,234.2)$ & $195.4(143.6,231.1)$ & $193.5(153.3,236.3)$ & 0.953 \\
Border zone-I [\%] & $2.10(1.3,3.7)$ & $1.35(0.4,2.2)$ & $2.64(2.1,4.3)$ & 0.001 \\
Border zone-II [\%] & $7.13(5.0,11.7)$ & $7.88(4.8,15.2)$ & $6.89(5.0,10.1)$ & 0.68 \\
Border zone-III [g] & $2.09(0.5,7.4)$ & $2.69(0.3,7.4)$ & $2.09(0.6,7.5)$ & 0.883 \\
\hline
\end{tabular}

Values as median ( $1^{\text {st }}$ quatile, $3^{\text {rd }}$ quartile); MVT — monomorphic ventricular tachycardia; LVEF — left ventricular ejection fraction; SA — surface area; LV - left ventricular

All 47 patients had BZ analysis performed by all 3 methods. Table 2 shows a comparison of BZ quantification as described in this study (BZ-I), in Yan et al. [14] (BZ-II), and in Schmidt et al. [18] (BZ-III). Border zone was significantly different between the non-inducible and MVT EPS groups using BZ-I (1.4\% vs. $2.6 \%, \mathrm{p}=0.001)$, but not using BZ-II (7.9\% vs. $6.9 \%, \mathrm{p}=0.68)$ or BZ-III (2.7 g vs. $2.1 \mathrm{~g}$, $\mathrm{p}=0.88)$. The relationship of $\mathrm{BZ}$ by each method to infarct size is demonstrated in Figure 3.

Several multivariate logistical regression models were evaluated, as shown in Table 3 . The first model included both infarct size and BZ-I (model 1), which demonstrated that BZ-I was the only significant predictor (OR 1.84 per \% change, $95 \%$ CI $1.03-3.28, \mathrm{p}=0.04)$. When including LVEF, infarct size and BZ-I (model 2), BZ-I was again the only independent predictor of EPS outcome (OR 2.07 per $\%$ change, $95 \%$ CI $1.08-3.98, \mathrm{p}=0.03)$. In a separate model that included BZ-I and infarct surface area (model 3), the confidence intervals for the OR for BZ-I (OR 1.71, 95\% CI 0.98-3.01, p = 0.06) in- cluded 1 , but is still consistent with the value of this parameter as a predictor of EPS outcome.

Another multivariate logistical regression model that included infarct size and BZ-II demonstrated that BZ-II was not a predictor of EPS outcome (OR 0.95 per \% change, 95\% CI $0.85-1.07, \mathrm{p}=$ $=0.395)$. When including both infarct size and LVEF in the model, BZ-II was still not a predictor (OR 0.95 per \% change, $95 \%$ CI $0.85-1.06, p=0.378)$. In model 4, which included BZ-I, BZ-II and BZ-III, only BZ-I was a significant, independent predictor of EPS outcome (OR 2.74 per \% change, 95\% CI 1.34-5.58, $\mathrm{p}=0.006)$.

Another logistical regression model was performed that included LVEF, infarct size and BZ-III. This analysis demonstrated that only infarct size was an independent predictors of EPS outcome, and BZ-III was not (OR 0.93 per \% change, $95 \% \mathrm{CI}$ $0.78-1.11, p=0.41)$. In a model that included only BZ-III and infarct size, BZ-III again was not a significant predictor (OR 0.92 per \% change, $95 \% \mathrm{CI}$ $0.77-1.10, \mathrm{p}=0.35)$. 


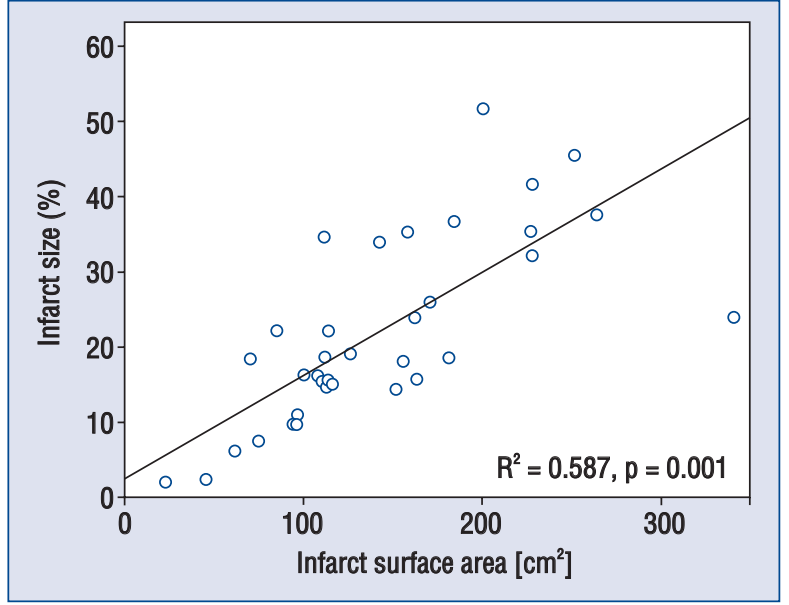

Figure 2. Infarct size (\%left ventricular mass) vs. infarct surface area $\left[\mathrm{cm}^{2}\right]$.

Table 3. Univariate and selected mutivariate analyses.

\begin{tabular}{|c|c|c|}
\hline LGE variable & OR (95\% CI) & $\mathbf{P}$ \\
\hline \multicolumn{3}{|l|}{ Univariate } \\
\hline Age & $1.04(0.98-1.12)$ & 0.216 \\
\hline History of $\mathrm{CHF}$ & $0.43(0.09-1.95)$ & 0.274 \\
\hline Gender & $0.11(0.02-0.65)$ & 0.015 \\
\hline Infarct size & $1.07(1.01-1.14)$ & 0.026 \\
\hline Border zone-I & $2.10(1.21-3.65)$ & 0.008 \\
\hline Border zone-II & $0.95(0.85-1.05)$ & 0.310 \\
\hline Border zone-III & $0.97(0.83-1.14)$ & 0.748 \\
\hline LVEF & $0.95(0.89-1.01)$ & 0.095 \\
\hline \multicolumn{3}{|l|}{ Multivariate } \\
\hline \multicolumn{3}{|l|}{ Model 1} \\
\hline Border zone-I & $1.84(1.03-3.28)$ & 0.040 \\
\hline Infarct size & $1.04(0.97-1.11)$ & 0.270 \\
\hline \multicolumn{3}{|l|}{ Model 2} \\
\hline Border zone-I & $2.07(1.08-3.98)$ & 0.029 \\
\hline Infarct size & $1.01(0.94-1.09)$ & 0.749 \\
\hline LVEF & $0.94(0.86-1.02)$ & 0.158 \\
\hline \multicolumn{3}{|l|}{ Model 3} \\
\hline Border zone-I & $1.71(0.98-3.01)$ & 0.061 \\
\hline Surface area & $1.00(1.00-1.00)$ & 0.076 \\
\hline \multicolumn{3}{|l|}{ Model 4} \\
\hline Border zone-I & $2.74(1.34-5.58)$ & 0.006 \\
\hline Border zone-II & $0.82(0.65-1.04)$ & 0.098 \\
\hline Border zone-III & $0.88(0.72-1.08)$ & 0.216 \\
\hline \multicolumn{3}{|l|}{ Model 5} \\
\hline Gender & $0.16(0.02-1.09)$ & 0.061 \\
\hline Border zone-I & $1.63(0.93-2.84)$ & 0.087 \\
\hline
\end{tabular}

$\mathrm{OR}$ - odds ratio; $\mathrm{Cl}$ - confidence interval; $\mathrm{CHF}$ - congestive heart failure; LVEF — left ventricular ejection fraction
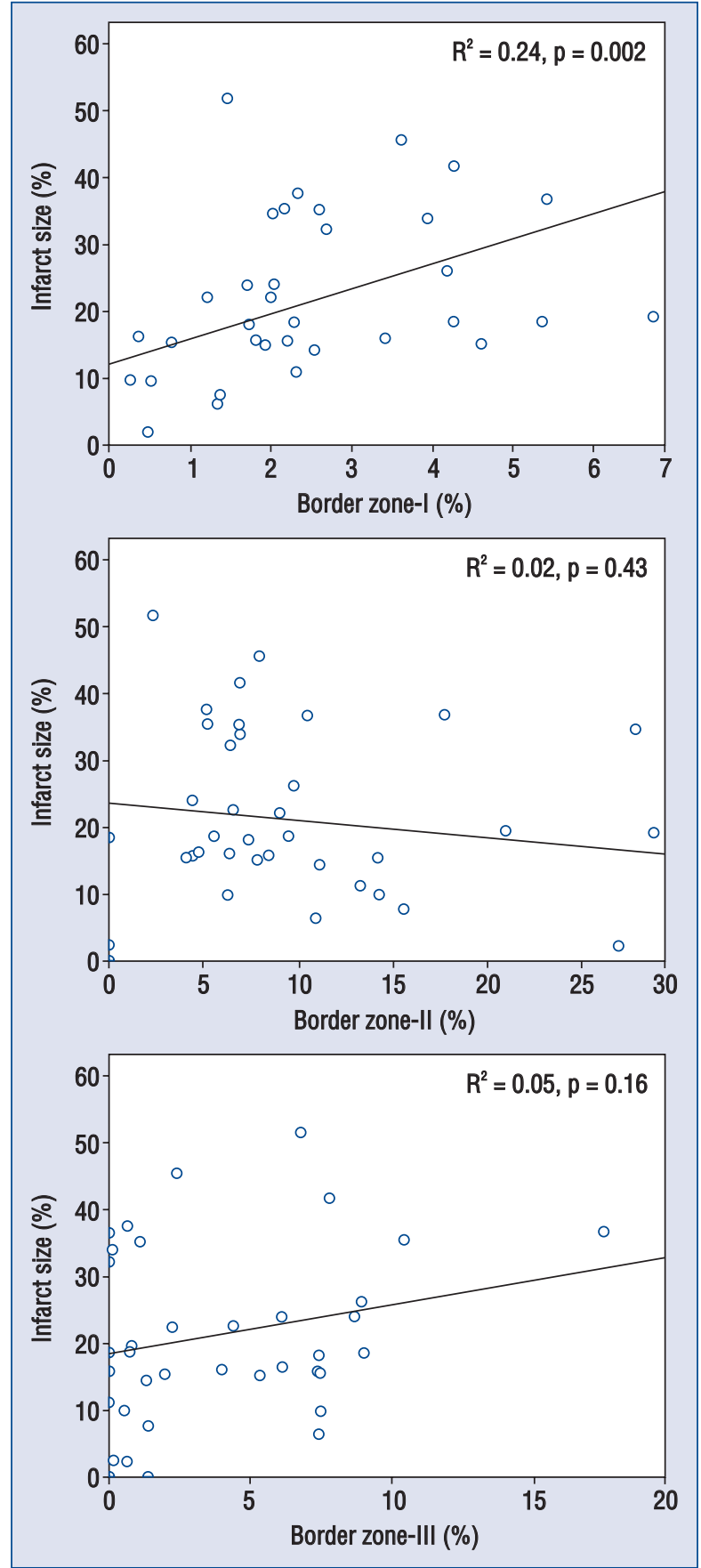

Figure 3. Comparison of infarct size vs. the three different methods of borderzone quantification. From the top to the botton: Border zone as a percent of left ventricular mass mass (border zone-l), Border zone as a percent of infarct size (border zone-II), and border zone mass by full-width half-max method (border zone-III).

In order to examine if $\mathrm{BZ}$ had a simply largely geometric relation to infarct size and surface area or was a unique measurement, its correlation with other infarct characteristics was evaluated. As shown in Figure 2, the original measurements of 
infarct size and surface area were highly correlated $\left(\mathrm{R}^{2}=0.587, \mathrm{p}<0.001\right)$. However, as demonstrated in Figure 3, the correlation of BZ-I $\left(\mathrm{R}^{2}=0.24\right.$, $\mathrm{p}=0.002)$, BZ-II $\left(\mathrm{R}^{2}=0.02, \mathrm{p}=0.43\right)$, and BZ-III $\left(R^{2}=0.05, p=0.16\right)$ to infarct size was poor. The correlation of BZ-I $\left(\mathrm{R}^{2}=0.19, \mathrm{p}=0.007\right), \mathrm{BZ}-\mathrm{II}$ $\left(\mathrm{R}^{2}=0.06, \mathrm{p}=0.13\right)$, and BZ-III $\left(\mathrm{R}^{2}=0.08, \mathrm{p}=0.08\right)$ to infarct surface area was also poor. The correlation among the different $\mathrm{BZ}$ measurements was also poor (BZ-I vs. BZ-II: $\mathrm{R}^{2}=0.02, \mathrm{p}=0.44$; BZ-I vs. BZ-III: $\mathrm{R}^{2}=0.003, \mathrm{p}=0.77$; BZ-II vs. BZ-III: $\mathrm{R}^{2}=$ $=0.083, \mathrm{p}=0.08)$.

\section{Long-term outcomes}

While not powered for outcome data, long-term data were available for the original study participants. Average follow-up was $1383.8 \pm 617.0$ days. There were 14 deaths, 6 in the non-inducible group (30.0\%), 2 in the PVT and VF group (22.2\%), and 6 in the MVT group (33.3\%). There was no significant difference in mortality among the groups. Cause of death was not known for all patients. There were 26 ICD implants (4 in the non-inducible group, 5 in the PVT/VF group and 17 in the MVT group). Five patients received appropriate ICD therapy for spontaneous ventricular arrhythmias (1 in the noninducible group, 4 in the MVT group).

Border zone was not significantly different between patients alive vs. dead by any method; BZ-I ( $1.9 \%$ vs. $2.4, \mathrm{p}=0.6)$, BZ-II (6.9\% vs. $9.4 \%, \mathrm{p}=0.3$ ) or BZ-III (2.3 g vs. $1.4 \mathrm{~g}, \mathrm{p}=0.6$ ). When age was controlled for, neither EPS outcome nor BZ was predictive of death or a composite endpoint of death or appropriate ICD therapy.

\section{Discussion}

The principle finding of this study is that $\mathrm{BZ}$ quantified by three different techniques on the same set of CMRs provides dramatically different results. The measurements of BZ-I, BZ-II, and BZ-III are not highly correlated with each other and the relationship with total infarct mass markedly differs so that BZ-II is inversely related to infarct mass while BZ-I and BZ-III are directly related. Furthermore, while BZ-I is an independent predictor of induction of MVT during EPS in this patient cohort, neither BZ-II nor BZ-III were. Further studies will be required to determine which of these measurements provides the most realistic physiologic measurement of the $\mathrm{BZ}$, and which provides optimal prognostic information.

Ventricular tachycardia commonly arises from reentry around a fixed lesion, such as an infarct scar
$[21,27]$. The critical region for these VT has been shown histologically to be bundles of surviving myocytes in the immediate peri-infarct area - the border zone [21]. Intracardiac electrical mapping studies have confirmed that the peri-infarct area is typically involved in VT [28-31], and catheter-based mapping and ablation of these areas has been shown to be an effective therapy for VT [32]. Slow conduction, a required component of reentrant arrhythmias, is found in the border of healing infarcts [33, 34]. In addition, areas of patchy infarct that may provide the geometric substrate for reentry [15], as well as areas of mixed dead and viable myocardium [21] have all been identified in the peri-infarct zone.

There are various clinical tests to attempt to identify patients with this substrate for ventricular arrhythmias. Some tests identify anatomic features associated with the peri-infarct border zone - for example, infarct size might be expected to be related to the mass of $\mathrm{BZ}$ present. As infarct size, until recently, could not be accurately measured, LVEF has been used as a surrogate risk marker. Although many factors influence LVEF [35, 36], in general, larger infarcts are associated with lower LVEF. As this has been a readily clinically available tool, it has been extensively studied and shown to be a useful predictor of SCD [37, 38]. LGE measurement of infarct scar by delayed-enhancement with gadolinium is a more specific measure of infarct size, allowing for direct quantification of the infarct morphology, which may be linked to arrythmogenesis [11]. EPS is a non-anatomic risk stratification tool that examines the electrical properties of the ventricle and identifies the presence of substrate for VT. BZ measurement, however, has the potential to be a direct, non-invasive measure of the anatomic substrate which has previously been only histologically defined.

While the data for the use of $\mathrm{BZ}$ as measured by LGE to predict cardiovascular risk appear promising, there are significant limitations in our understanding of this imaging finding. While histologic data have confirmed that the peri-infarct zone is an area of abnormal electrophysiologic properties [33], it is not proven that this is what is being represented by the intermediate-intensity pixels near or within a LGE identified infarct. Ideally, all of these pixels would be due to areas that are an admixture of viable and non-viable myocardium, which may be a substrate for arrhythmias. However, these pixels may also occur due to other imaging conditions and artifacts. Partial volume effects, where normal myocardium, blood pool, or epicardial fat may exist within the same voxel volume along with the myo- 
cardial infarct, can produce intermediate-intensity values. This may produce the appearance of $\mathrm{BZ}$ on LGE imaging, even if the actual tissue is not an admixture of viable and non-viable myocardium, and presumably not arrhythmogenic [39]. Partial volume can occur in the short axis plane along the border of the infarct, as well as in the $Z$ direction from above or below the infarct. In addition, poor T1 nulling of normal myocardium, as well as low signal-to-noise ratios in general, can increase the amount of intermediate-intensity pixels, and thereby falsely elevate the BZ levels [40]. However, despite all the potential confounders in the measurement of BZ by LGE, these data as well as prior studies [14], suggest it has clear biologic implications. In our data, BZ had a positive, but poor correlation with both infarct size as well as infarct surface area, suggesting that there is a minimal amount of artifact from peri-infarct volume averaging. Techniques that may minimize these other causes of intermediate-intensity pixels should help further improve the specificity of BZ to identify pro-arrhythmic substrate.

Contemporary literature on the use of LGE imaging for the quantification of infarct $\mathrm{BZ}$ reveals a number of statistical techniques for the measurement and reporting of $\mathrm{BZ}$ burden. We analyzed our images using these different techniques to look for similarities and differences, as shown in Table 2. One technique was reported in a recent study by Yan et al. [14]; a cohort of 144 patients with CAD had CMR images taken and were followed for an average of 2.4 years. Yan et al. [14] defined BZ (method II) as regions of signal intensity between 2 and 3 SD above normal myocardium, indexed to infarct size (pixels with values $3 \mathrm{SD}$ above). In this cohort, BZ-II was a univariate predictor of total mortality $(\mathrm{p}=0.01)$ and cardiovascular mortality $(\mathrm{p}=0.009)$, as was LVEF $(\mathrm{p}=0.01)$ and LV endsystolic volume index $(\mathrm{p}=0.002)$. A multivariable model showed that BZ-II and LV end-systolic volume index were the 2 strongest, independent predictors of all-cause and cardiovascular mortality. While these data demonstrate a link between BZ and mortality, little is known about the selection or actual cause of death for these patients, who exhibited a higher than expected mortality rate of $20 \%$ over 2.4 years. The mechanism for death was conjectured to be reentrant ventricular arrhythmias.

In the present study, we quantified $\mathrm{BZ}$ as reported by Yan et al. [14], but also as a percent of the total LV mass, in a similar fashion as infarct size has been reported in the past. Reporting BZ as a percentage of the infarct size [14] may discard important information about the gross amount of ar- rhythmic BZ tissue. In both Yan's report and in this study, when BZ is indexed to infarct size (method II) as opposed to LV mass (method I), there is an inverse relationship between $\mathrm{BZ} \%$ and infarct size, as patients with smaller infarcts have larger BZ to infarct size ratios. A patient with a relatively small amount of arrhythmic BZ tissue, with a very small infarct, would have a large reported $\mathrm{BZ}$ ratio by method II. This inverse relationship of $\mathrm{BZ}$ and infarct size in method II does not correlate to the observation that larger infarcts are more arrhytmogenic [11]. We felt that a more useful metric for $\mathrm{BZ}$ would take into account the total amount of arrhythmic substrate present, and should have a positive correlation with total infarct \%. This lead to method I, reporting BZ as indexed to total LV mass.

Another technique for $\mathrm{BZ}$ calculation has been reported by Schmidt et al. [18]. This study examined a cohort of 47 patients with CAD prior to ICD implant for primary prevention of sudden death. These patients underwent CMR, as well as either EPS or non-invasive programmed stimulation to look for ventricular inducibility. They defined BZ (method III) as pixels greater than the peak value in the normal myocardium but less than $50 \%$ of the highest signal in scar tissue (visually defined). This was reported unadjusted, in grams of myocardium. In this cohort, BZ-III was a significant predictor of inducibility of MVT ( $p=0.015)$, but not total infarct size.

The comparison of techniques in this study does not establish the superiority of one method over another; rather, it highlights the variety of reporting methods currently being employed for $\mathrm{BZ}$ quantification, and that they differ significantly. All 3 methods described are similar in that they are predictive of either mortality or EPS outcomes. This is likely due to a common shared underlying physiology that they indirectly or directly measure - be it actual histologic $\mathrm{BZ}$, or some relation to infarct mass or surface area. Until histologic confirmation of $\mathrm{BZ}$ is available and is related to a particular CMR technique, this issue will likely remain ambiguous.

\section{Limitations of the study}

A significant limitation of this study is that it is non-randomized and utilizes a small sample size. With only 18 patients in the MVT group, the multivariate analysis can be of limited statistical accuracy, and should be considered only as thought provoking and requiring additional study. However, the univariate results should still be valid despite the small sample and of significant importance. In addition, EPS inducibility identifies the presence of 
substrate for VT, but may not be an adequate surrogate for arrhythmic sudden death. Finally, this CMR dataset was acquired from 1998-2002, and the image quality is somewhat less than with modern techniques. However, this only underscores how the different $\mathrm{BZ}$ algorithms respond diversely to varying imaging artifacts in a clinical dataset.

\section{Conclusions}

This study provides additional information in the understanding of the mechanism by which $\mathrm{BZ}$ is associated with enhanced cardiovascular mortality. Previously, inducible MVT during EPS has been shown to be highly predictive of both sudden death as well as total mortality [22, 41]. Our data provide a confirmation of the link between BZ quantification and arrhythmias in a human cohort. We also demonstrated that $\mathrm{BZ}$ is not simply a surrogate measurement of infarct size or infarct surface area based on geometric relationships, but contains additional data. Border zone is a more specific predictor of inducible arrhythmia than LVEF, so has the potential for being an improved predictor of mortality due to SCD. Although no such relationship on outcome was identified in this study, this may be related to the limited sample size. Further studies are warranted, both to correlate imaging findings of $\mathrm{BZ}$ to histology, and to assess the prognostic significance of infarct characteristics such as infarct size and BZ.

Author's contributions: JR designed study, created software for border zone analysis, performed analysis, and drafted manuscript. DL and EW conceived of study, assisted with the border zone analysis and manuscript creation. DB executed the original prospective study and data collection from which a large portion of this retrospective study was based on. AK, RP, and JG helped refine the study goals, and assisted with manuscript creation. All authors read and approve the final manuscript.

Conflict of interest: JR: None, EW: None, AK: None, DB: None, RP: None, JG: None, DL: Consulting fees from Siemens (modest) and St. Jude Medical (modest). Research funding from the American Heart Association (significant) and St. Jude Medical (significant).

\section{References}

1. Zheng Z-J, Croft JB, Giles WH, Mensah GA. Sudden cardiac death in the United States, 1989 to 1998. Circulation, 2001; 104: 2158-2163.
2. Bardy GH, Lee KL, Mark DB et al. Amiodarone or an implantable cardioverter-defibrillator for congestive heart failure. NEJM, 2005; 352: 225-237.

3. Buxton AE, Lee KL, Fisher JD et al. A Randomized study of the prevention of sudden death in patients with coronary artery disease. NEJM, 1999; 341: 1882-1890.

4. Moss AJ, Hall WJ, Cannom DS et al. Improved survival with an implanted defibrillator in patients with coronary disease at high risk for ventricular arrhythmia. NEJM, 1996; 335: 1933-1940.

5. Moss AJ, Zareba W, Hall WJ et al. Prophylactic implantation of a defibrillator in patients with myocardial infarction and reduced ejection fraction. NEJM, 2002; 346: 877-883.

6. Zipes DP, Camm AJ, Borggrefe M et al. ACC/AHA/ESC 2006 Guidelines for management of patients with ventricular arrhythmias and the prevention of sudden cardiac death: A report of the American College of Cardiology/American Heart Association Task Force and the European Society of Cardiology Committee for Practice Guidelines (Writing Committee to Develop Guidelines for Management of Patients With Ventricular Arrhythmias and the Prevention of Sudden Cardiac Death): Developed in Collaboration With the European Heart Rhythm Association and the Heart Rhythm Society. Circulation, 2006; 114: e385-e484.

7. Buxton AE. Identifying the high risk patient with coronary artery disease; is ejection fraction all you need? J Cardiovasc Electrophysiol, 2005; 16: S25-S27.

8. Goldberger JJ, Cain ME, Hohnloser SH et al. American Heart Association/American College of Cardiology Foundation/Heart Rhythm Society Scientific Statement on noninvasive risk stratification techniques for identifying patients at risk for sudden cardiac death. A scientific statement from the American Heart Association Council on Clinical Cardiology Committee on Electrocardiography and Arrhythmias and Council on Epidemiology and Prevention. Circulation, 2008; 118: 1179-1199.

9. The Antiarrhythmics versus Implantable Defibrillators (AVID) Investigators. A comparison of antiarrhythmic-drug therapy with implantable defibrillators in patients resuscitated from near-fatal ventricular arrhythmias. NEJM, 1997; 337: 1576-1584.

10. Mahrholdt H, Wagner A, Holly TA et al. Reproducibility of chronic infarct size measurement by contrast-enhanced magnetic resonance imaging. Circulation, 2002; 106: 2322-2327.

11. Bello D, Fieno DS, Kim RJ et al. Infarct morphology identifies patients with substrate for sustained ventricular tachycardia. J Am Coll Cardiol, 2005; 45: 1104-1108.

12. Kwong RY, Chan AK, Brown KA et al. Impact of unrecognized myocardial scar detected by cardiac magnetic resonance imaging on event-free survival in patients presenting with signs or symptoms of coronary artery disease. Circulation, 2006; 113: 2733-2743.

13. Wu E, Ortiz JT, Tejedor P et al. Infarct size by contrast enhanced cardiac magnetic resonance is a stronger predictor of outcomes than left ventricular ejection fraction or end-systolic volume index: Prospective cohort study. Heart, 2007: hrt.2007.122622.

14. Yan AT, Shayne AJ, Brown KA et al. Characterization of the peri-infarct zone by contrast-enhanced cardiac magnetic resonance imaging is a powerful predictor of post-myocardial infarction mortality. Circulation, 2006; 114: 32-39.

15. Bolick DR, Hackel DB, Reimer KA, Ideker RE. Quantitative analysis of myocardial infarct structure in patients with ventricular tachycardia. Circulation, 1986; 74: 1266-1279.

16. Jones-Collins BA, Patterson RE. Quantitative measurement of electrical instability as a function of myocardial infarct size in the dog. Am J Cardiol, 1981; 48: 858-863. 
17. Wilber DJ, Lynch JJ, Montgomery D et al. Postinfarction sudden death: Significance of inducible ventricular tachycardia and infarct size in a conscious canine model. Am Heart J, 1985; 109: 8-18.

18. Schmidt A, Azevedo CF, Cheng A et al. Infarct tissue heterogeneity by magnetic resonance imaging identifies enhanced cardiac arrhythmia susceptibility in patients with left ventricular dysfunction. Circulation, 2007; 115: 2006-2014.

19. Ashikaga H, Mickelsen SR, Ennis DB et al. Electromechanical analysis of infarct border zone in chronic myocardial infarction. Am J Physiology, Heart Circulation Physiology, 2005; 289: H1099-H1105.

20. Hu Q, Wang X, Lee J et al. Profound bioenergetic abnormalities in peri-infarct myocardial regions. Am J Physiology, Heart Circulation Physiology, 2006; 291: H648-H657.

21. de Bakker JM, van Capelle FJ, Janse MJ et al. Reentry as a cause of ventricular tachycardia in patients with chronic ischemic heart disease: Electrophysiologic and anatomic correlation. Circulation, 1988; 77: 589-606.

22. Denniss AR, Richards DA, Cody DV et al. Prognostic significance of ventricular tachycardia and fibrillation induced at programmed stimulation and delayed potentials detected on the signal-averaged electrocardiograms of survivors of acute myocardial infarction. Circulation, 1986; 74: 731-745.

23. Kim RJ, Wu E, Rafael A et al. The Use of contrast-enhanced magnetic resonance imaging to identify reversible myocardial dysfunction. NEJM, 2000; 343: 1445-1453.

24. Wu E, Judd RM, Vargas JD, Klocke FJ, Bonow RO, Kim RJ. Visualisation of presence, location, and transmural extent of healed Q-wave and non-Q-wave myocardial infarction. Lancet, 2001; 357: 21-28.

25. Simonetti O, Kim R, Fieno D. An improved MR imaging technique for the visualization of myocardial infarction. Radiology, 2001; 218: 215-223.

26. Rubenstein JC, Ortiz JT, Wu E et al. The use of periinfarct contrast-enhanced cardiac magnetic resonance imaging for the prediction of late postmyocardial infarction ventricular dysfunction. Am Heart J, 2008; 156: 498-505.

27. Bakker JM, van Capelle FJ, Janse MJ et al. Slow conduction in the infarcted human heart. 'Zigzag' course of activation. Circulation, 1993; 88: 915-926.

28. Costeas C, Peters NS, Waldecker B, Ciaccio EJ, Wit AL, Coromilas J. Mechanisms causing sustained ventricular tachycardia with multiple QRS morphologies: Results of mapping studies in the infarcted canine heart. Circulation, 1997: 96: 3721-3731.
29. Gardner PI, Ursell PC, Fenoglio JJ Jr., Wit AL. Electrophysiologic and anatomic basis for fractionated electrograms recorded from healed myocardial infarcts. Circulation, 1985; 72: 596-611.

30. Peters NS, Coromilas J, Severs NJ, Wit AL. Disturbed connexin 43 gap junction distribution correlates with the location of reentrant circuits in the epicardial border zone of healing canine infarcts that cause ventricular tachycardia. Circulation, 1997; 95: 988-996.

31. Peters NS, Wit AL. Myocardial Architecture and ventricular arrhythmogenesis. Circulation, 1998; 97: 1746-1754.

32. Stevenson WG, Friedman PL, Kocovic D, Sager PT, Saxon LA, Pavri B. Radiofrequency catheter ablation of ventricular tachycardia after myocardial infarction. Circulation, 1998; 98: 308-314.

33. Ursell PC, Gardner PI, Albala A, Fenoglio JJ Jr., Wit AL. Structural and electrophysiological changes in the epicardial border zone of canine myocardial infarcts during infarct healing. Circulation Res, 1985; 56: 436-451.

34. Yao J-A, Hussain W, Patel P, Peters NS, Boyden PA, Wit AL. Remodeling of gap junctional channel function in epicardial border zone of healing canine infarcts. Circulation Res, 2003; 92: 437-443.

35. Bassand JP, Bassand JP. Left ventricular remodelling after acute myocardial infarction: Solved and unsolved issues. Eur Heart J, 1995; 16 (suppl. I): 58-63.

36. Sonnenblick EH, Sonnenblick EH. Correlation of myocardial ultrastructure and function. Circulation, 1968; 38: 29-44.

37. Bigger JT, Jr., Fleiss JL, Kleiger R, Miller JP, Rolnitzky LM. The relationships among ventricular arrhythmias, left ventricular dysfunction, and mortality in the 2 years after myocardial infarction. Circulation, 1984; 69: 250-258.

38. Sanz G, Castaner A, Betriu A et al. Determinants of prognosis in survivors of myocardial infarction: A prospective clinical angiographic study. NEJM, 1982; 306: 1065-1070.

39. Klocke FJ, Wu E, Lee DC. "Shades of gray" in cardiac magnetic resonance images of infarcted myocardium: Can they tell us what we'd like them to? Circulation, 2006; 114: 8-10.

40. Wagner A, Mahrholdt $\mathrm{H}$, Thomson L et al. Effects of time, dose, and inversion time for acute myocardial infarct size measurements based on magnetic resonance imaging-delayed contrast enhancement. J Am Coll Cardiol, 2006; 47: 2027-2033.

41. Bourke JP, Richards DA, Ross DL, Wallace EM, McGuire MA, Uther JB. Routine programmed electrical stimulation in survivors of acute myocardial infarction for prediction of spontaneous ventricular tachyarrhythmias during follow-up: results, optimal stimulation protocol and cost-effective screening. J Am Coll Cardiol, 1991; 18: 780-788. 